\title{
UN CAMINO HACIA LA INCLUSION
}

\author{
Liliana Lopez Morales \\ lilopez@poligran.edu.co \\ Maria Alexandra Vergez Madiedo \\ mavergez@poligran.edu.co \\ Institución Universitaria Politécnico Grancolombiano \\ Licenciatura en Educación para la Primera Infancia. \\ Colombia
}

\section{Resumen}

Este trabajo se realizó con el fin de analizar la situación de los estudiantes y las instituciones educativas frente a la inclusión. En el desarrollo de la investigación se puede conocer el proceso de inclusión de un niño con necesidades educativas especiales y como la institución ha ido transformado su desarrollo curricular con el fin de brindarle todas las condiciones esenciales para su crecimiento a nivel personal, social e intelectual; con repercusiones que lleguen a los espacios de educación inicial. Este trabajo se realizó durante un lapso de ocho meses (marzo-noviembre de 2019), en la cual se recolecto información pertinente y necesaria sobre el estudiante, la institución y especialistas externos quienes estaban apoyando la inclusión.

La inclusión es de anotar que debe permitir el aprendizaje y la participación de todos los estudiantes que puedan sentirse excluidos por alguna necesidad educativa especial o por alguna forma diferente de aprender. Es así, que la institución objeto genera espacios para recibir niños que han estado apartados del sistema escolar por diversas circunstancias, encontrando allí una enseñanza diferente con posibilidades para los niños, pero esto a la vez debe servir para generar una nueva estructuración de los distintos planes escolares, el PEl, el manual de convivencia donde se muestre la manera en que se realiza la atención de todos los estudiantes sin exclusiones, así mismo es necesario determinar la cantidad de estudiantes con alguna necesidad especial que puedan tener ya que un mayor número significaría contar con un manejo con personal de apoyo dentro de los salones de clases además de las modificaciones estructurales que eso implicaría.

Palabras clave: estudiante, educación especial, acceso a la educación, aprendizaje, integración escolar 


\section{INTRODUCCIÓN}

En el manejo de la temática relacionada con la inclusión es elemental conocer distintas definiciones que van ligadas a este concepto. En primera instancia es necesario definir la educación como un derecho humano en el cual el Estado, los adultos (padres y docentes) y los niños son la base fundamental, ya sea procurando por brindarlo de la mejor manera u obteniendo sus beneficios.

Al intentar incluir a alumnos y alumnas con necesidades especiales dentro de establecimientos regulares sin los recursos adecuados, que van desde la infraestructura, apoyo profesional especializado y un curriculum flexible, provocan una discriminación "negativa" dentro de los propios establecimientos, lo que finalmente producen una deserción, ahora a causa de la incomprensión que sientes niños y niñas con necesidades diversas dentro del espacio escolar. (Claro, 2007, p.181)

En el marco de la creación de estrategias para contrarrestar estos obstáculos el Ministerio de Educación Nacional estableció unos lineamientos con el fin de "entregar orientaciones y herramientas que permitan consolidar desde las mismas secretarías de educación una gestión basada en la inclusión, la equidad y la calidad del servicio educativo para estas poblaciones" (Ministerio de Educación Nacional, 2005, p. 5).

Con relación a lo expuesto, se puede observar que la inclusión es una solución viable a estos inconvenientes que presentan en el ámbito educativo y social, guiada por el Estado y puesta en práctica en los colegios con el apoyo de toda la comunidad educativa (E. Norman-Acevedo, 2018).

"La "inclusión" o la "educación inclusiva" no es otro nombre para referirse a la integración del "Alumnado con Necesidades Educativas Especiales". Implica un enfoque diferente para identificar e intentar resolver las dificultades que surgen en las escuelas." (Booth y Ainscow, 2000, p. 18).

\section{Método}

Para iniciar se hizo una indagación en la biografía del estudiante a nivel familiar, escolar y de salud, para luego continuar con la consulta dentro de la institución educativa sobre el proceso de inclusión llevado a cabo con el estudiante (Elisondo \& Donolo, 2015). Luego se indaga sobre las leyes que promueven la inclusión para lograr implementarla por medio de la comunicación paralela y la revisión de material dentro de la institución educativa como lo son el manual de convivencia y el PEI, con el fin de presentar estrategias que permitan la actuación dentro del marco legal, en favor de los diferentes estudiantes con necesidades educativas especiales (Daza-Orozco, 2015 y 2019).

En este proyecto se utilizó la investigación en acción la cual debe posibilitar la interacción con otros maestros y una autorreflexión sobre cómo se está llevando a cabo la enseñanza y como a partir de esas experiencias vividas en clase se pueden generar nuevas posibilidades las cuales favorecerán a los estudiantes y a su vez enriquecerán la labor docente(Oviedo \& Silva, 2017).

En resumen, las características más destacadas del profesional «amplio» son: una capacidad para un autodesarrollo profesional autónomo mediante un sistemático autoanálisis, el estudio 
de la labor de otros profesores y la comprobación de ideas mediante procedimientos de investigación en el aula. (Stenhouse, 1991, p. 197).

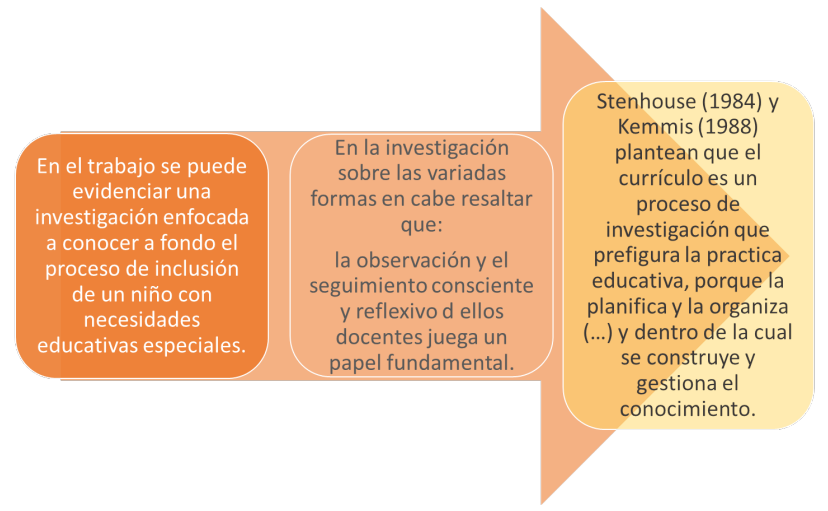

\section{Resultados}

Los principales resultados de la investigación se encaminan hacia una inclusión real tanto para el estudiante estudiado como para los que lleguen a la institución en busca de una oportunidad.

Luego de la investigación se observó que la institución decidió encaminar los procesos hacia las normativas existentes en temas relacionados con la inclusión. La institución educativa en su ánimo de llevar a cabo la inclusión ha empezado a tener una asesoría por parte de una empresa externa la cual brinda toda la información necesaria en cuanto a términos institucionales y legales para el ingreso o permanencia de niños con necesidad de inclusión.
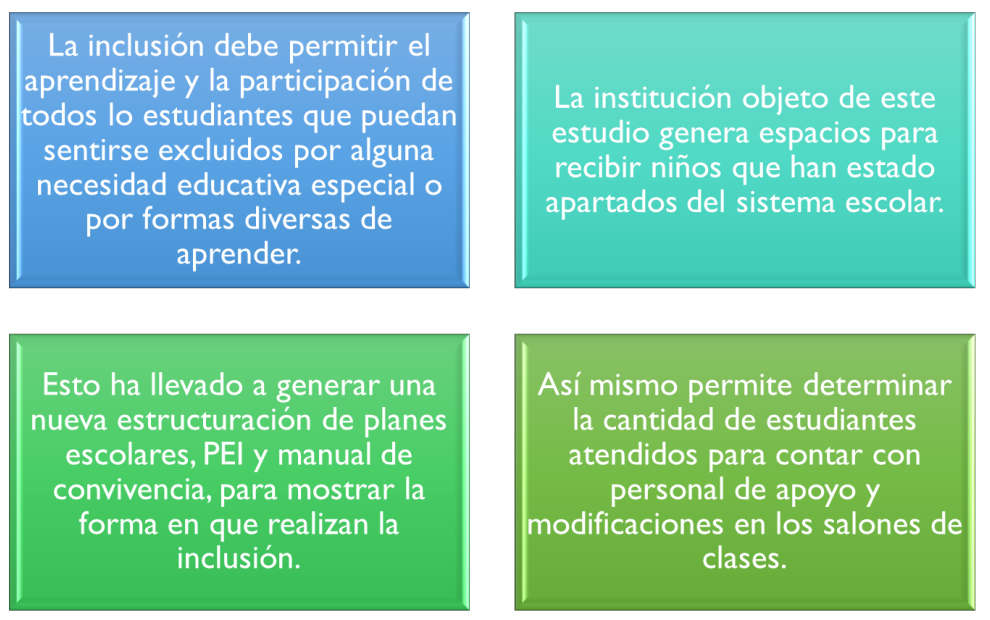

\section{Discusión y Conclusión}

El termino inclusión cada vez se hace más común, debido a que se ha ampliado el concepto de aprendizaje y en la actualidad hay muchos factores que son considerados para brindar las mejores condiciones de este, contemplando las múltiples diferencias de raza, credo, cultura, capacidades y otras. Por ende el Ministerio de Educación ha instaurado leyes que cuidan lo mencionado anteriormente, haciendo que cada vez sean mayores planteles educativos los que la contemplen. Sin embargo, la cultura de Latinoamérica aún es muy excluyente y el incluir hace parte de un camino de educar y educarnos abriendo nuestra percepción de la inclusión y lo que realmente se requiere para 
que esta sea efectiva y verdadera (Dos Santos Amaro Elías; Nogales Bocio, Antonia Isabel; Pérez

Calle, Begoña; Pérez Lagos, Camila; Marta-Lazo, Carmen; Barredo Ibáñez, Daniel; De La Garza

Montemayor, Daniel Javier; Murcia Quiñones, Harvey; Gil Quintana, J, Jenny Juliana, 2019).

América Latina se caracteriza por altos niveles de iniquidad, exclusión y fragmentación social.

Pese a la gran expansión de la educación y los esfuerzos realizados, todavía persisten

desigualdades educativas en función de los distintos estratos socioeconómicos, culturas y

características individuales del alumnado, como consecuencia del modelo homogeneizador de

los sistemas educativos. (Machado, 2000, p.5)

En la actualidad, la inclusión más que un tema a tener en cuenta se convierte en una necesidad presente en cada institución, sea pública o privada. Este proceso de la inclusión invita a la educación a dar un cambio, donde los procedimientos cuantitativos se varíen por procedimientos cualitativos que reconozcan el desarrollo más que el resultado.

Es por eso por lo que, al tomar conciencia de la inclusión, surge la necesidad de contar con unos orientadores pedagógicos con una capacidad más receptiva y flexible para conocer un contexto, unas necesidades inmediatas y brindar el acompañamiento que se requiera según los diferentes niveles desempeño que pueden surgir. (Ainscow, 2001).

\section{Referencias bibliográficas}

Ainscow, M. (2001). Comprendiendo el desarrollo de las escuelas inclusivas. Reino Unido: Facultad de Educación de Manchester. Booth, T. y Ainscow, M. (2000). Index for Inclusion. Developing learning and participation in schools. [índice para la inclusión. El desarrollo del aprendizaje y la participación en las escuelas]. London: Centre for Studies on Inclusive Education, CSIE.

Claro, J.P. (2007). Estado y desafíos de la inclusión educativa en las regiones Andina y Cono Sur. REICE - Revista Electrónica Iberoamericana sobre Calidad, Eficacia y Cambio en Educación. 5 (5e), 179-187.

Daza-Orozco, CE. (2019). Iniciación científica: conceptualización, metodologías y buenas prácticas. Bogotá: Institución Universitaria Politécnico Grancolombiano. Retrieved from http://www.scoif.com/iniciacion-cientifical

Daza-Orozco, CE. (2019). Historia de la infancia en el cine colombiano. Bogotá. Institución Universitaria Politécnico Grancolombiano. Retrieved from http://alejandria.poligran.edu.co/handle/10823/1648

Daza Orozco, CE. (2015). Cartografía de los consumos y experiencias de la niñez en internet. Panorama, (11), 8 - 25. Recuperado a partir de https://revia.areandina.edu.co/index.php/Ll/article/view/438

Dos Santos Amaro Elías; Nogales Bocio, Antonia Isabel; Pérez Calle, Begoña; Pérez Lagos, Camila; Marta-Lazo, Carmen; Barredo Ibáñez, Daniel; De La Garza Montemayor, Daniel Javier; Murcia Quiñones, Harvey; Gil Quintana, J, Jenny Juliana, A. S. L. R. P. (2019). Ciudadanías digitales: perspectivas desde los medios, el periodismo y la educomunicación (1st ed.; C.-C. M. Isabel, ed.). Bogotá: Institución Universitaria Politécnico Grancolombiano.

Kemmis, S. (1988). El currículo más allá de la teoría de la reproducción. Madrid, España: Ediciones Morata.

Machado, A. (2000). Prólogo a la versión en castellano para América Latina y el Caribe. Index for Inclusion. Developing learning and participation in schools. [índice para la inclusión. El desarrollo del aprendizaje y la participación en las escuelas]. Londres, CSIE.

Ministerio de Educación Nacional (2005). Lineamientos de política para la atención educativa a poblaciones vulnerables. Población con necesidades educativas especiales.

Norman-Acevedo, E. (2018). Rompiendo Barreras: 10 Años de la Educación Virtual en el Politécnico Grancolombiano. (primera; Eduardo Norman-Acevedo, ed.). Retrieved from http://alejandria.poligran.edu.co/handle/10823/1146

Oviedo, L. B., \& Silva, M. C. (2017). LA INVESTIGACIÓN ACCIÓN Y EL APRENDIZAJE POR PROYECTOS EN EL MARCO DEL MODELO PEDAGÓGICO ENSEÑANZA PARA LA COMPRENSIÓN. EXPERIENCIA DEL COLEGIO VISIÓN MUNDIAL EN COMUNIDADES VULNERABLES DE MONTERÍA-Action research and Project Based Learning in ... Panorama, 11(21), 38-51. https://doi.org/10.15765/pnrm.v11i21.1053

Stenhouse, L. (1984). Investigación y Desarrollo del Currículo. Madrid, España: Ediciones Morata. 